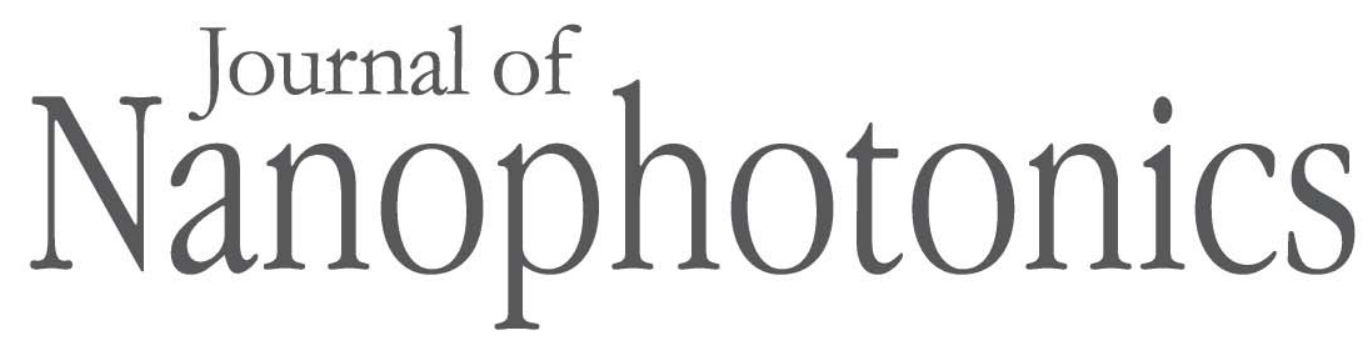

SPIEDigitalLibrary.org/jnp

\title{
Commentary: Optical nanoantennas: from communications to super-resolution
}

Diana C. Skigin

Marcelo Lester 


\title{
Commentary: Optical nanoantennas: from communications to super-resolution
}

\author{
Diana C. Skigin ${ }^{\mathrm{a}}$ and Marcelo Lester ${ }^{\mathrm{b}}$ \\ ${ }^{a}$ Universidad de Buenos Aires, Ciudad Universitaria, \\ Grupo de Electromagnetismo Aplicado and IFIBA (CONICET), \\ Departamento de Física, Facultad de Ciencias Exactas y Naturales, Pabellón I, \\ C1428EHA Buenos Aires, Argentina \\ dcs@df.uba.ar \\ ${ }^{\mathrm{b}}$ Universidad Nacional del Centro de la Provincia de Buenos Aires, Grupo Optica de \\ Sólidos-Elfo, Instituto de Física Arroyo Seco, Facultad de Ciencias Exactas, Pinto 399
} (cp 7000) Buenos Aires, Argentina

Paper 11062CTR received Apr. 29, 2011; revised manuscript received May 9, 2011; accepted for publication May 9, 2011; published online Jun. 1, 2011. [DOI: 10.1117/1.3595688]

The field of optical nanoantennas has recently experienced an extensive growth in research activities, owing to the fascinating possibility of confining electromagnetic radiation to subwavelength spatial domains via surface plasmon polaritons. As a result of nanoconfinements, the local electromagnetic field can be significantly enhanced, leading to a number of extraordinary effects. The potential of these devices is spectacular; applications range from plasmonic waveguides and nano-interconnects for ultrafast communications, to solar-cells energy-efficient guiding, biosensors, amplification of molecular responses, increased Raman scattering, and medical cancer treatments, among others.

An antenna is a part of a system designed to transmit or receive electromagnetic waves. This device is able to convert the radiation energy of the propagating wave into localized energy, and vice versa. The same energy conversion mechanism also occurs in metallic nanostructures at the optical range: the electromagnetic radiation can be resonantly coupled to the free electrons of noble metals, resulting in intense concentrations of energy at the surface of the nanostructure, in a localized way. This resonant phenomenon is known in the literature as surface plasmon resonance. Thus, a metallic nanostructure might operate like an optical antenna.

Following this concept, the simplest optical antenna is a metallic sphere of radius much smaller than the incident wavelength, tuned in its plasmon frequency. One of the first devices based on these ideas was proposed by Bailey and Fletcher, who patented an electromagnetic wave converter (1973), and possibly Lin and colleagues were the first to report in 1996 resonant light absorption by a fabricated subnanostructure and high frequency rectification in the visible range. ${ }^{1}$ Since then, thanks to the great advances in manufacturing systems with controlled nanoscale details, interest in these resonant devices has increased significantly. In recent years, the study and design of optical antennas has aroused a special interest in the scientific community. Unlike low-frequency antennas (radio and microwave), optical antennas need a specific design for each application. However, in most cases the basic physics underlying the device operation is the same: localized plasmon oscillations at the base of the antenna, which are transmitted through collective oscillations of the free electrons in the metallic nanostructure.

In general, an optical antenna interacts with the transmitter or the receiver only for a predesigned set of frequencies, i.e., in a discrete way like a quantum system, significantly enhancing the signal between the transmitter and the receiver. One of the simplest designs for these devices is formed by two interacting particles (dimer) and core-shell systems are probably the most promising dimers. These nanoparticles can be tuned by changing the geometry, such as the

$1934-2608 / 2011 / \$ 25.00$ @ 2011 SPIE 
thickness of the metal coating or the core, or by breaking its symmetry. More complex systems have also shown its effectiveness as nanoantennas: tips or tip arrays, metallic chains, or systems comprising both of them. ${ }^{2}$ All these configurations can be regarded as metamaterials or materials with average properties, which might exhibit magnetic behavior in the optical range or a negative refractive index, among other interesting properties. To illustrate the nanoantenna properties at resonance, in Fig. 1 we show the near field intensity and the far field pattern for a structure comprising six silver nanowire chains illuminated by an evanescent wave, at resonance. The direction of the main lobe in the far field pattern is that of the -3 diffraction order, and this direction can be controlled by appropriately choosing the geometrical parameters of the structure, such as the period and the inclination angle of each chain with respect to the periodicity direction. The maximum transmitted intensity in this compound array is an order of magnitude larger than that of a single chain with the same parameters, and also the directivity of the pattern is increased. Therefore, the periodic addition of chains to the structure not only improves the transmittance but also increases its directivity. The near field configuration evidences an enhancement even in the vicinity of the furthest cylinders of each chain.

Like radio frequency antennas, optical antennas are generally designed employing metallic structures. The main difference between them is that metals are excellent conductors at low frequencies while in the optical range the metallic losses are important, and surface plasmon propagation is attenuated significantly. This effect is noticeable in the power propagation in metallic chains as well as in flat interfaces, where the propagation length reaches several wavelengths. ${ }^{3,4}$

One of today's major technological challenges is the fabrication and characterization of these nanostructures with dimensions of several tens of nanometers, which can confine and

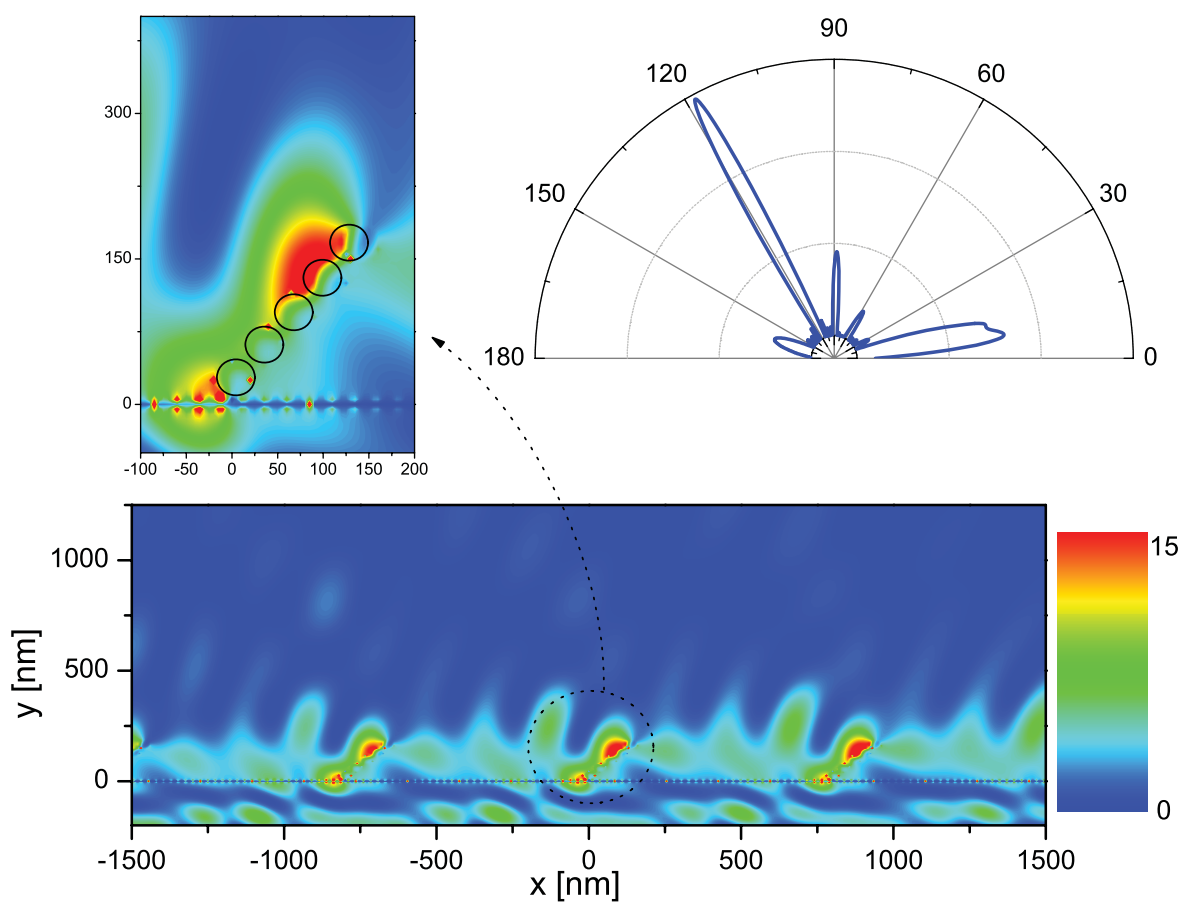

Fig. 1 Near field intensity and far field pattern for a nanoantenna made of an array of six finite chains of metallic wires distributed periodically. The system is illuminated by an evanescent wave generated by total internal reflection in the plane interface at $y=0$. In this example, the impinging wavelength is $\lambda=400 \mathrm{~nm}$ ( $p$ polarization, magnetic field parallel to the wires), which corresponds to the plasmon resonance of a single chain (inset). The inset shows an enlargement of the zone near one chain, which comprises five wires. The array is tilted $45^{\circ}$ with respect to the periodicity direction. The far field pattern shows that the direction of maximum reflection can be pre-designed at will by appropriately choosing the geometrical parameters of the structure. 
guide electromagnetic fields in sizes of the order of a tenth of a wavelength, i.e., well below the classical diffraction limit. The design of optical antennas is a recently developed area of research. These devices are far from being those well-known telescopic antennas (such as those of old cars and portable radios), directional or omni-directional microantennas like those of mobile phones or GPS systems, with which we are familiar. Optical antennas bring a conceptual jump.

In order to characterize these photonic structures, a number of theoretical and experimental approaches are currently being developed. Just as electronic circuits can be tuned by radio frequency antennas, these "photonic circuits" could be used to tune optical antennas in a wide range of the visible spectrum. 5,6

On the other hand, different configurations of optical antennas are being experimentally explored and characterized. Among the considered models there are the dipole-type antenna (with its morphological variants) and the bowtie type [a scheme of this type of antenna is shown in Fig. 2(a)]. ${ }^{7}$ There are more complex ones such as the Yagi-Uda type, consisting of an array of equally-spaced nano-rods [see Fig. 2(b)]. ${ }^{8}$ The main aim of the reasearch in this area pretends to manipulate, characterize, and predict the field concentration in reduced areas.

The development of ultrasmall spots of light may be the key to achieve, at the same time, a high spatial resolution and an efficient emitter-receiver system. Communication at these spatial distances is exclusively governed by evanescent waves. When these highly localized and very intense fields are activated, the optical properties in the gap between particles are altered, leading to interesting physical effects such as superluminal signal transmission, ${ }^{9}$ which, in turn, implies a conceptual change in communications. Given the characteristics of these resonators, optical antennas constitute a promising interface device between optical nanocircuits. ${ }^{10}$

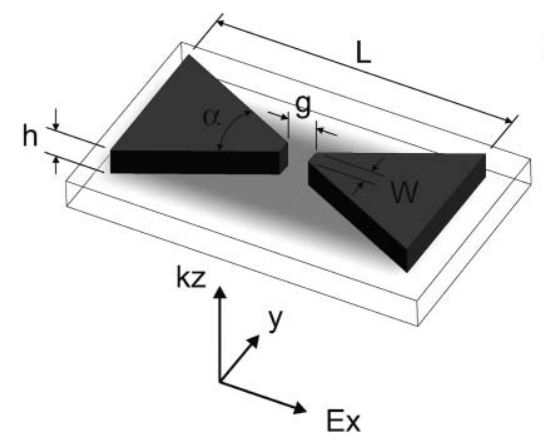

(a)



Fig. 2 Two plasmonic nano-optical antennas: (a) Bowtie type. The incident field propagates in the $z$ direction and is polarized in the $x$ direction. The near field is intensified just in the gap $g$ and in the triangles' corners; (b) Yagi type antenna of 5 elements. The typical distance between the antenna elements is about $\lambda / 4$. Variations of the geometrical parameters and materials make it possible to change the optical operation range. 
The potential for ultrafast communications is not the only possible use of these devices. In general, the plasmon excitation is very sensitive to the medium surrounding the metal nanoparticle as well as to the particle shape. These features of the system itself make optical antennas an exceptional probe to explore the complex biological world in vitro or in vivo. One of the great challenges in biology is to understand the complex molecular dynamics in its natural environment. For this purpose, a small amount of organic matter should be scanned (below $50 \mathrm{~nm}$ ), and these lengths are precisely of the order of magnitude that an optical antenna can provide for the field confinement. Thus, it is possible to explore individual cells and to obtain their topography, biochemical maps, and fluorescence images at the same time, with nanometer details. Besides, other techniques based on optical or plasmon resonance antennas are being proposed and used in biology and medical physics, for example, using these resonators as fluorescent markers or as an ablation mechanism of cancer cells. ${ }^{11,12}$

The first steps are given. The cellular super-resolution and the generation of optical circuits seem to be just around the corner helped by optical antennas.

\section{Acknowledgments}

The authors gratefully acknowledge partial support from Consejo Nacional de Investigaciones Científicas y Técnicas CONICET (PIP 112-200801-01880 and PIP 114-200900-0145). D.S. gratefully acknowledges partial support from Universidad de Buenos Aires (UBA X208). M.L. is grateful to Universidad Nacional del Centro de la Provincia de Buenos and Instituto de Física Arroyo Seco and to to Mr. Santiago C for his cooperation.

\section{References}

1. G. H. Lin, R. Abdu, and J. O'M. Bockris, "Investigation of resonance light absorption and rectification by subnanostructures," J. Appl. Phys. 80(1), 565-568 (1996).

2. M. Lester and D. C. Skigin, "An optical nanoantenna made of plasmonic chain resonators," J. Opt. 13, 035105 (2011).

3. S. A. Maier, M. L. Brongersma, and H. A. Atwater, "Electromagnetic energy transport along arrays of closely spaced metal rods as an analogue to plasmonic devices," Appl. Phys. Lett. 78, 16-18 (2001).

4. X-X. Liu and A. Alu, "Subwavelength leaky-wave optical nanoantennas: Directive radiation from linear arrays of plasmonic nanoparticles," Phys. Rev. B 82, 144305 (2010).

5. A. Alu and N. Engheta, "Input impedance, nanocircuits loading, and radiation tuning of optical nanoantennas," Phys. Rev. Lett. 101, 043901 (2008).

6. L. Novotny, "Effective wavelength scaling for optical antennas," Phys. Rev. Lett. 98, 266802 (2007).

7. H. Fischer and O. J. F. Martin, "Engineering the optical response of plasmonic nanoantennas," Opt. Express 16, 9144 (2008).

8. T. H. Taminiau, F. D. Stefani, and N. F. van Hulst, "Enhanced directional excitation and emission of single emitters by a nano-optical Yagi-Uda antenna," Opt. Express 16, 1085810861 (2008).

9. G. Nimtz, "Superluminal signaling by photonic tunneling," IEEE J. Sel. Top. Quantum Electron. 9, 79-85 (2003).

10. A. Alu and N. Engheta, "Wireless at the nanoscale: Optical interconnects using matched nanoantennas," Phys. Rev. Lett. 104, 213902 (2010).

11. N. Tadaaki, G. Han, Ch. Hoang, J. Wi, A. Pucci, D. Weber, F. Neubrech, V. M. Silkin, D. Enders, O. Saito, and M. Rana, "Plasmons in nanoscale and atomic-scale systems," Sci. Technol. Adv. Mater. 11, 054506 (2010).

12. M. F. Garcia-Parajo, "Optical antennas focus in on biology," Nat. Photon. 2, 201-203 (2008). 